Review Article

\title{
Different Forms of ER Stress in Chondrocytes Result in Short Stature Disorders and Degenerative Cartilage Diseases: New Insights by Cartilage-Specific ERp57 Knockout Mice
}

\author{
Yvonne Rellmann $(\mathbb{D}$ and Rita Dreier $(\mathbb{D}$ \\ Institute of Physiological Chemistry and Pathobiochemistry, Waldeyerstraße 15, 48149 Münster, Germany \\ Correspondence should be addressed to Rita Dreier; dreierr@uni-muenster.de
}

Received 30 August 2018; Accepted 13 November 2018; Published 17 December 2018

Guest Editor: Tomasz Poplawski

Copyright (C) 2018 Yvonne Rellmann and Rita Dreier. This is an open access article distributed under the Creative Commons Attribution License, which permits unrestricted use, distribution, and reproduction in any medium, provided the original work is properly cited.

\begin{abstract}
Cartilage is essential for skeletal development by endochondral ossification. The only cell type within the tissue, the chondrocyte, is responsible for the production of macromolecules for the extracellular matrix (ECM). Before proteins and proteoglycans are secreted, they undergo posttranslational modification and folding in the endoplasmic reticulum (ER). However, the ER folding capacity in the chondrocytes has to be balanced with physiological parameters like energy and oxygen levels. Specific cellular conditions, e.g., a high protein demand, or pathologic situations disrupt ER homeostasis and lead to the accumulation of poorly folded or misfolded proteins. This state is called ER stress and induces a cellular quality control system, the unfolded protein response (UPR), to restore homeostasis. Different mouse models with ER stress in chondrocytes display comparable skeletal phenotypes representing chondrodysplasias. Therefore, ER stress itself seems to be involved in the pathogenesis of these diseases. It is remarkable that chondrodysplasias with a comparable phenotype arise independent from the sources of ER stress, which are as follows: (1) mutations in ECM proteins leading to aggregation, (2) deficiencies in ER chaperones, (3) mutations in UPR signaling factors, or (4) deficiencies in the degradation of aggregated proteins. In any case, the resulting UPR substantially impairs ECM protein synthesis, chondrocyte proliferation, and/or differentiation or regulation of autophagy and apoptosis. Notably, chondrodysplasias arise no matter if single or multiple events are affected. We analyzed cartilage-specific ERp57 knockout mice and demonstrated that the deficiency of this single protein disulfide isomerase, which is responsible for formation of disulfide bridges in ECM glycoproteins, is sufficient to induce ER stress and to cause an ER stress-related bone phenotype. These mice therefore qualify as a novel model for the analysis of ER stress in chondrocytes. They give new insights in ER stress-related short stature disorders and enable the analysis of ER stress in other cartilage diseases, such as osteoarthritis.
\end{abstract}

\section{Cartilage Enables Skeletal Development, Bone Growth, and Diarthrodial Joint Function}

Cartilage is a connective tissue with essential functions in embryonic development and throughout life. During bone development by endochondral ossification, cartilaginous templates of future bones are formed and later gradually replaced by bone $[1,2]$. The bone formation starts with the generation of cartilage condensations, consisting of prechondrogenic mesenchymal cells. These cells differentiate into chondrocytes, produce a cartilage-specific ECM, and build bone templates ( $=$ bone anlagen), in which the central chondrocytes start to proliferate and progressively differentiate into metabolically highly active hypertrophic chondrocytes. During proliferation and hypertrophic differentiation, chondrocytes produce large amounts of extracellular proteins that form structural components of the ECM or act as local growth factors. Through a variety of these growth factors, the chondrocytes trigger the differentiation of osteoblasts from the 
surrounding periosteum to form the bone collar [3]. In addition, vascular invasion is initiated and brings osteoblasts and osteoclasts into this so-called primary ossification center. These cells replace the cartilage by bone through removal of cartilaginous extracellular matrix and deposition of newly formed trabecular bone. Most hypertrophic chondrocytes die by apoptosis. However, recently, it was proven that some of the osteoblasts arise from hypertrophic chondrocytes by transdifferentiation [4]. All processes recur in the secondary ossification centers in both epiphyses of the long bones. Following ossification in the primary and secondary ossification centers, cartilaginous tissue remains on the bone surfaces as articular cartilage, where it is responsible for frictionless movement of the joints. Cartilage also remains in the growth plates between the ossification centers, where it is responsible for long bone growth until the growth plate fuses during puberty. Lengthening of a bone is dependent on proliferation and maturation of chondrocytes and the increasing production and secretion of ECM molecules by the chondrocytes. However, the largest contribution comes from a dramatic increase in the volume of hypertrophic chondrocytes in the growth plate as they undergo terminal differentiation [5]. Any imbalance between proliferation and hypertrophy can lead to skeletal defects and in particular to chondrodysplasias [6]. Chondrodysplasias comprise several hundred distinct forms of skeletal diseases from severe disorders that are perinatal lethal to milder conditions that are recognized postnatally [7]. The latter are characterized by a disproportionate short stature, eye abnormalities, cleft palate, and hearing loss [8]. In addition to skeletal development and bone growth, cartilage is involved in the maintenance and function of diarthrodial joints.

\section{A Proper ER Function Is a Prerequisite for Effective Protein Synthesis and Secretion by Chondrocytes}

To build and maintain cartilage, a proper function of the endoplasmic reticulum (ER) is essential in chondrocytes, as they are responsible for the production of large amounts of ECM proteins during skeletal development and growth. Due to avascularity of cartilage, the secretory chondrocytes experience a variety of stresses, such as low oxygen tension and limited nutrient conditions $[9,10]$, and consequently, the protein folding capacity in the cells has to be balanced with physiological parameters like energy and oxygen levels [11]. However, different cellular conditions, e.g., phases of high protein demand or pathologic situations, prevent ER homeostasis and lead to the accumulation of poorly folded proteins. This physiological or pathological state is called ER stress and induces a cellular quality control system, the so-called unfolded protein response (UPR), an adaptive mechanism to cope with ER stress to restore homeostasis [12].

Prior to their secretion, all proteins destined not only for the extracellular space but also for the plasma membrane or for secretory compartments undergo posttranslational modification, folding, and maturation in the rough ER (Figure 1) [13]. For this purpose, the lumen of the ER contains resident molecular chaperones, protein disulfide isomerases (PDIs), and folding factors that multiply the rate of protein folding. The folding complexes are active in a specific environment of high $\mathrm{Ca}^{2+}$ concentration and oxidizing conditions $[12,14]$. The chaperones and folding enzymes can be assigned to different protein families: (1) members of the heat shock family (e.g., BiP, GRP94), (2) serpins (e.g., HSP47), (3) lectins (e.g., calreticulin, calnexin, and EDEM), (4) oxidoreductases or protein disulfide isomerases (e.g., PDI, ERp57), and (5) peptidyl-prolyl cis/trans isomerases (cyclophilins, FK506-binding proteins, and parvulin-like peptidyl-prolyl cis/trans isomerases) [14-16]. Initially, the proteins to be folded are targeted to the ER by hydrophobic signal sequences that are cotranslationally recognized by signal recognition particles. After transition through a translocon complex in the ER membrane, the signal peptide is cleaved off by signal peptidases in the ER lumen and the nascent polypeptide is posttranslationally modified, e.g., by the oligosaccharyltransferase which is responsible for $\mathrm{N}$-linked glycosylation. Attachment of carbohydrate moieties, called glycans, to asparagine within the Asn-X-Ser/Thr consensus sequence enhances the intrinsic solubility of nascent polypeptides during folding, potentially by masking hydrophobic patches, but also allows critical interactions with the lectin chaperones calnexin and calreticulin [17].

Via particular domains at their N-termini, the lectins calnexin and calreticulin specifically bind monoglycosylated $\mathrm{N}$-linked glycans on the nascent proteins, after these were attached by the oligosaccharyltransferase and trimmed by glycosidases I and II and ER mannosidases [18]. The Ntermini of calnexin and calreticulin in addition bind the PDI ERp57, assisting in folding by disulfide exchange reactions. Like other protein disulfide isomerases in the ER, ERp57 is responsible for correct disulfide bridge formation. After a first round of folding, calnexin or calreticulin releases the protein and glucosidase II removes the final glucose molecule from its glycan, thereby inhibiting the binding of lectin chaperones again. However, until the folding process is not completed, a uridine diphosphate-glucose-glycoprotein transferase adds a new glucose molecule to the glycan again, and the protein enters the calnexin/calreticulin cycle for a second time allowing another round of folding. Such glycosylation-folding reglycosylation cycles continue until the native conformation is finally achieved or proteins aggregate due to misfolding $[12,19]$. This review focusses on ERp57, that is, as a part of the calnexin/calreticulin cycle, mainly engaged in folding of glycoproteins with unstructured disulfide-rich domains [20,21]. An overview about these and other distinct functional roles of ERp57 in various cellular compartments playing a role under physiological and pathological conditions is given elsewhere [22].

Correctly folded proteins move via vesicular transport to the Golgi apparatus. There are additional modifications such as O-glycosylation occur, and sorting of the proteins into different kinds of vesicles is established to enable a further transport to different cellular compartments or 

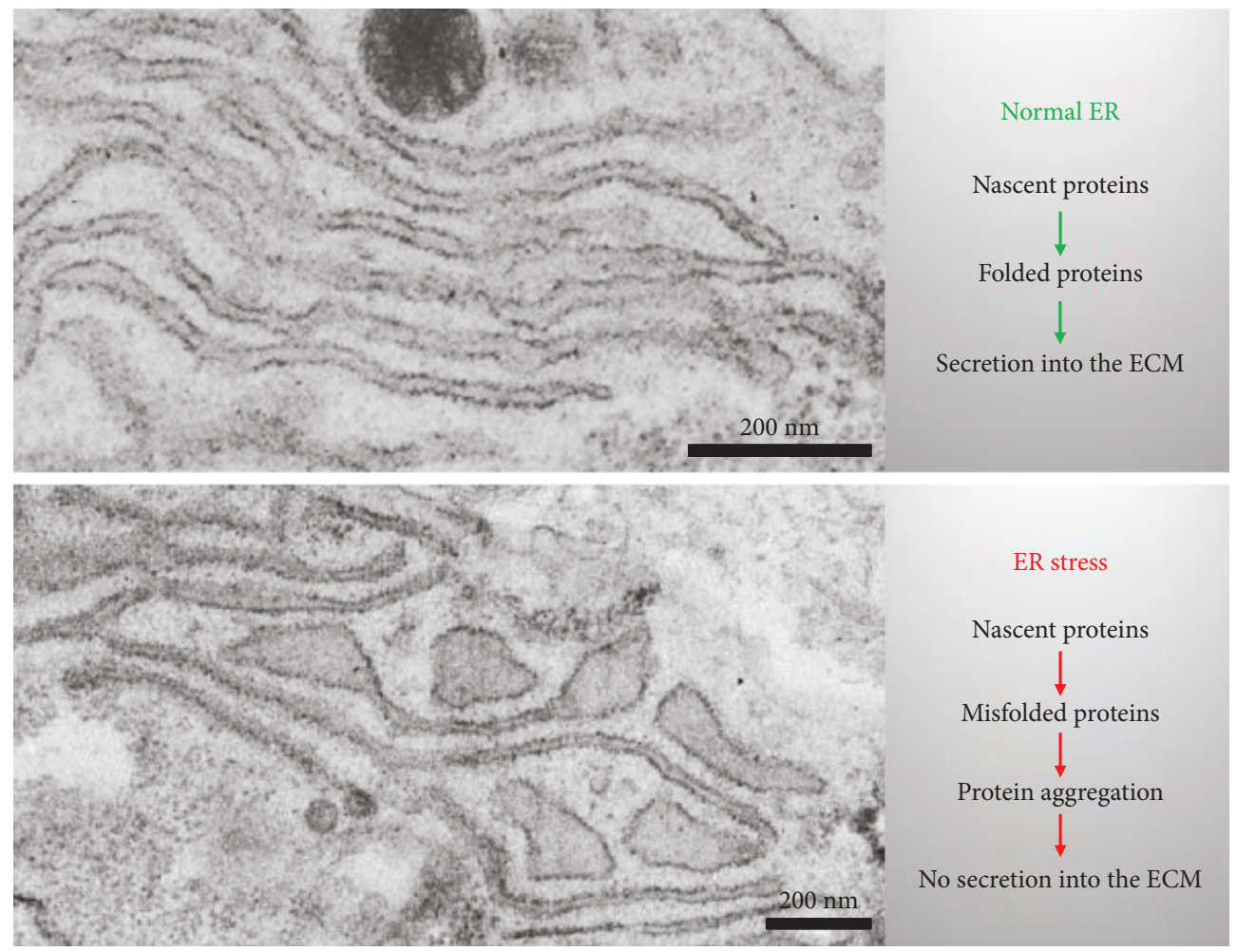

FIGURE 1: ER stress induces morphological and functional changes in chondrocytes. Normal chondrocytes produce large amounts of cartilage components. Before secretion into the ECM, these proteins undergo posttranslational modification and folding in the ER. If these processes fail, ER stress arises and misfolded proteins aggregate in the ER. This leads to a dilation of ER cisternae and a diminished protein secretion into the ECM.

secretion into the extracellular space. In case of incorrect folding of ECM proteins or protein overload in the ER, unfolded or misfolded proteins accumulate in the ER and subsequently activate the UPR. This complex quality control system leads to a general stop of cellular protein synthesis, an increased production of additional chaperones and other folding proteins, and to the degradation of aggregated proteins by ER-associated degradation (ERAD type I) or autophagy (ERAD type II). ERAD type II represents an autophagic pathway in which soluble and insoluble misfolded proteins are incorporated in autophagosomes, which then fuse with lysosomes. ERAD type I targets soluble misfolded proteins only. These are polyubiquitinylated and translocated into the cytosol, where they are degraded in the proteasome [23]. However, when the combined efforts of UPR and ERAD do not readjust cellular homeostasis, cell death by apoptosis is initiated, in order to enable general tissue homeostasis. [24].

\section{The UPR Initiates a Stepwise Rescue System for ER-Stressed Cells}

The adaptive UPR comprises three parallel signaling pathways starting from ER stress sensor proteins located in the ER membrane: ATF6 $\alpha$ (activating transcription factor 6 alpha), IRE1 $\alpha$ (inositol-requiring enzyme 1 alpha), and PERK (protein kinase RNA-like endoplasmic reticulum kinase) [25-27]. At the luminal side of the ER membrane, BiP (immunoglobulin heavy-chain-binding protein), also known as glucose-regulated protein 78 (GRP78), binds to these sensor proteins and keeps them inactive. Upon binding of $\mathrm{BiP}$ to unfolded or misfolded proteins accumulating in the $\mathrm{ER}, \mathrm{BiP}$ is released from the ER stress sensors, which trigger the UPR signaling pathways. On the first route, $\mathrm{BiP}$-free ATF6 $\alpha$ traffics to the Golgi apparatus, where it is processed by the site 1 and site 2 proteases (S1P and S2P). The released ATF $6 \alpha$ fragment acts as a transcription factor, enters the nucleus, and induces UPR genes encoding additional chaperones or initiators of ERAD [28]. On the second pathway, BiP-free IRE1 $\alpha$ is activated by oligomerization and autophosphorylation [29]. Active IRE1 $\alpha$ degrades certain mRNAs through regulated IRE1-dependent decay (RIDD) [30] and induces splicing of the transcription factor XBP1 (X-box-binding protein 1). The spliced transcription factor $\mathrm{XBP} 1 \mathrm{~s}\left(\mathrm{XBP} 1_{\text {spliced }}\right)$ then directly activates gene expression for folding proteins and quality control mechanisms in the ER. On the third route, oligomerized and autophosphorylated PERK acts as a kinase on eIF2a (eukaryotic translation initiation factor 2A) and thereby stops global transcription, thus reducing the overall protein synthesis and decreasing the load of unfolded proteins in the ER [31]. However, due to preferential translation of mRNAs containing short open reading frames in the $5^{\prime}$ UTRs, the amount of transcription factor ATF4 is 
increased. ATF4 positively regulates the expression of UPR genes that are involved in amino acid metabolism, antioxidant response, folding, and regulation of autophagy and apoptosis. Examples of such ATF4-induced genes are CHOP (C/EBP homologous protein) and GADD34 (growth arrest and DNA damage-inducible 34) [32].

\section{ER Stress in Cartilage Is Important under Physiological and Pathological Conditions}

One should consider that ER stress or UPR signaling pathways play a crucial role in chondrocytes in phases of high protein synthesis, e.g., during bone development by endochondral ossification. As cartilage is a nonvascularized tissue, low energy levels and hypoxic conditions prevail. ER stress, therefore, is essential for normal differentiation and hypertrophic maturation of chondrocytes under these tough, but physiological conditions [33, 34].

In addition, ER stress is triggered by pathological conditions, such as metabolic dysfunction, $\mathrm{Ca}^{2+}$ ion imbalances, and expression of mutant proteins, or inducible by specific drugs. Under all circumstances, the direct consequence of ER stress is the initiation of UPR signaling in order to return to cellular homeostasis. However, this is not always possible, and thus, cellular imbalances occur that lead to ER stressrelated pathological outcomes. Due to unresolved ER stress in chondrocytes, diseases of the skeletal system, such as chondrodysplasias, arise.

\section{Various Mouse Models with ER Stress in Chondrocytes Display Phenotypes Resembling Skeletal Diseases Associated with Growth Plate Abnormalities and Dwarfism}

Several whole-body knockout mouse models demonstrate the general necessity of a proper protein folding in the ER for developmental processes, organ function, and cellular homeostasis. Homozygous deletion of ER chaperones such as calreticulin, BiP, GRP94, ERp57, or UDP-glucoseglycoprotein glucosyltransferase results in embryonic lethality $[14,35]$. Thus, the function of these proteins is exclusive and essential for embryonic development. Here, we focus on the impact of chondrocytes to the development and growth of long bones. Different actions of these cells are important for endochondral ossification, such as the finely tuned proliferation and maturation, the raising production and secretion of specific ECM molecules, the increase in the volume of hypertrophic chondrocytes, and the exact regulation of chondrocyte death by apoptosis at the lower end of the epiphyseal plate. If one or more of these processes fail, skeletal development is impaired and short stature diseases, like chondrodysplasias, may develop.

In order to specifically analyze the role of ER stress in chondrocytes and its relevance to chondrodysplasias, more and more mouse models with cartilage-specific changes in protein folding have been developed (Table 1) [36, 37]. From these mice, one can deduce that prolonged ER stress, e.g., due to a mutation of an ECM protein initiating poor folding and aggregation in the ER, is a pathogenic mechanism behind short stature diseases like metaphyseal chondrodysplasia type Schmid (MCDS), multiple epiphyseal dysplasia (MED), or pseudoachondrodysplasia (PSACH). Similarly, mice with mutations in proteins of the ER folding machinery [38], mutations in UPR signaling factors [39, 40], or mutations in proteins of the secretory and degradative pathways eliminating aggregated proteins display related skeletal phenotypes. This substantiates that ER stress acts as a pathogenic factor in chondrodysplasias.

To get a well-defined overview about mouse models with ER stress in chondrocytes, one should discriminate between (1) transgenic mice with mutations in genes encoding ECM proteins, (2) transgenic mice with mutations in genes encoding exogenous proteins that are normally not expressed in cartilage, (3) mice with a knockout of genes encoding proteins of the ER folding machinery, (4) mice with a knockout of genes of UPR signaling factors, (5) mice with a knockout of proteins involved in the degradation of aggregated proteins, and (6) mice with a knockout of proteins essential for protein trafficking and secretion.

5.1. Transgenic Mice with Mutations in Genes Encoding Cartilage ECM Proteins. The extracellular matrix of cartilage is composed of a set of self-assembled secreted macromolecules that form a dynamic network of fibrillar and nonfibrillar structures. The key macromolecules of cartilage ECM are collagens II, IX, and XI, forming collagen fibrils and thus are mainly responsible for the tensile strength; proteoglycans, primarily aggrecan, responsible for the osmotic swelling and elastic properties; noncollageneous glycoproteins such as COMP and matrilins, connecting various ECM components; and hyaluronan, providing compression strength, lubrication, and hydration within the cartilaginous ECM [41]. Due to mutations in genes encoding such ECM proteins, misfolding may occur during its synthesis, which initiates ER stress. The following mouse models demonstrate that ER stress seems to be critically involved in the development of skeletal diseases, as all mice display chondrodysplasialike phenotypes, no matter which deficiency they have.

5.1.1. Mouse Model of Chondrodysplasia Associated with a Mutation in the Col2a1 Gene (p.Gly1170Ser in Col2a1). Mutations in the $\alpha 1$ chain of procollagen type II initiate chondrodysplasias of different severity from lethal to mild forms [42]. Such disease-inducing mutations often occur in the triple-helical domain (Gly-X-Y domain) of collagen II alpha 1 chains and initiate intracellular retention of the targeted protein with induction of ER stress and activation of UPR signaling [37]. One prominent example is a knockin mouse model harboring a col2alp.Gly1170Ser mutation, in which the growth plate develops abnormally because chondrocytes undergo apoptosis before hypertrophy. This leads to the disappearance of hypertrophic zones. The detailed investigation of this mouse model suggested that this early chondrocyte death is related to the ER stress-UPR-apoptosis cascade and that this is the main cause of the p.Gly1170Ser-induced chondrodysplasia in mice and men [43]. 


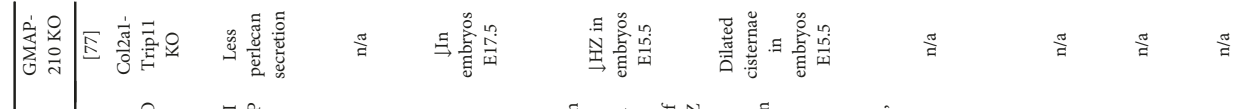

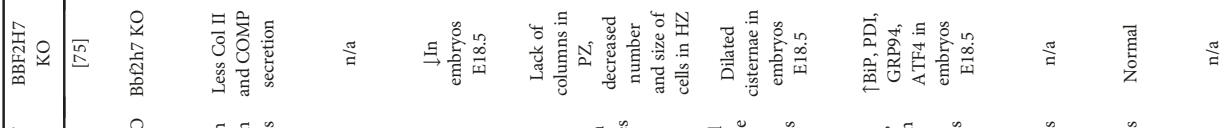

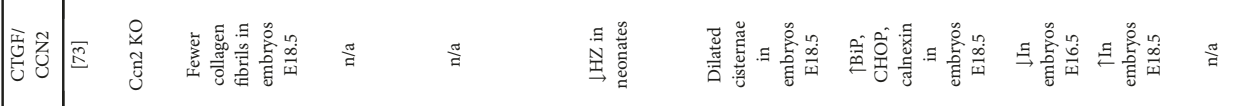

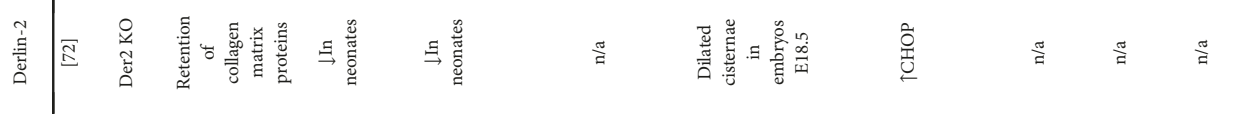

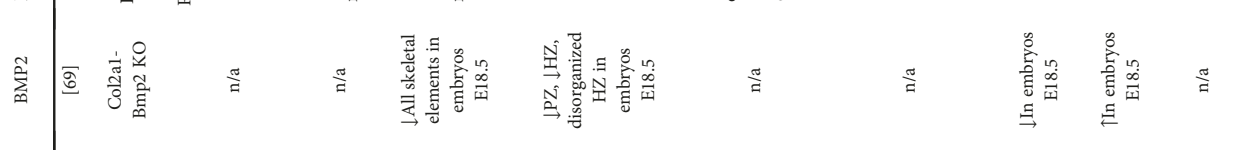

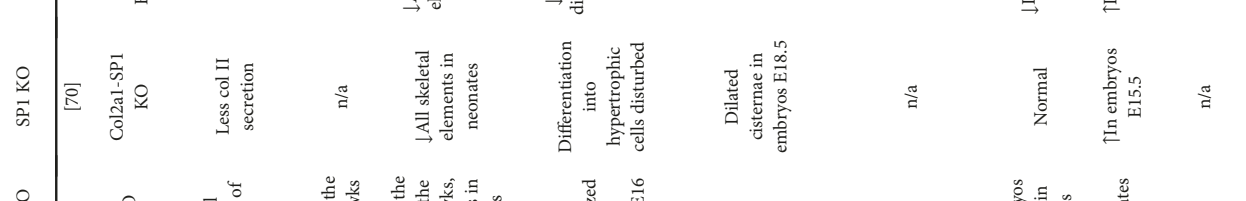

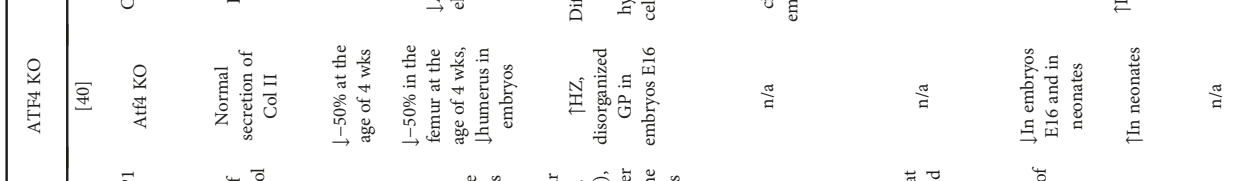

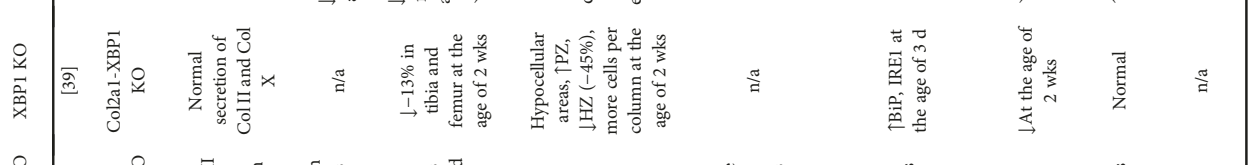

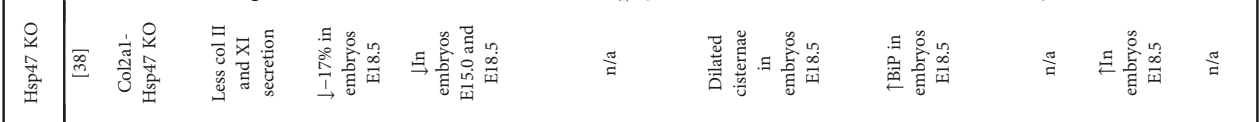

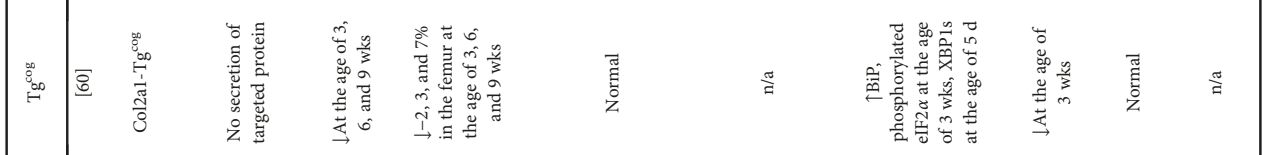

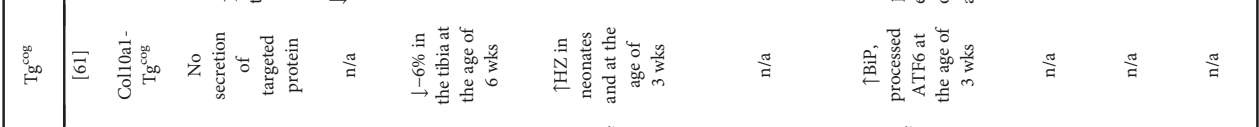

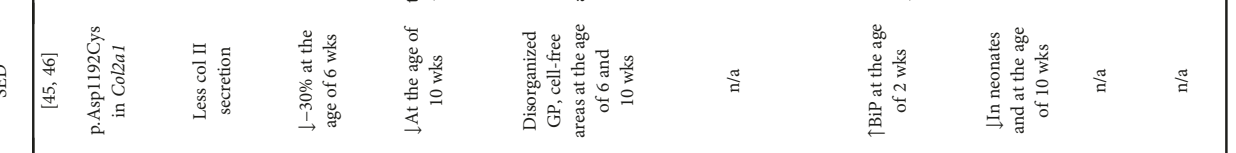

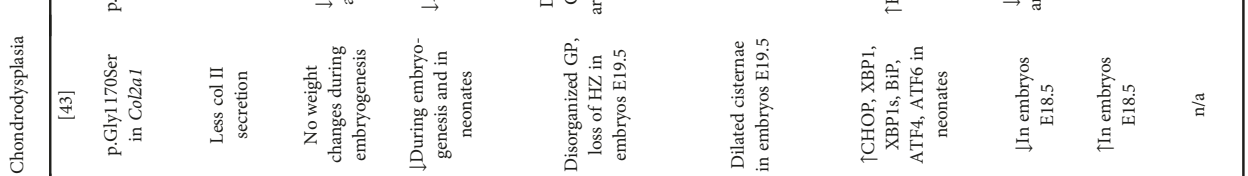

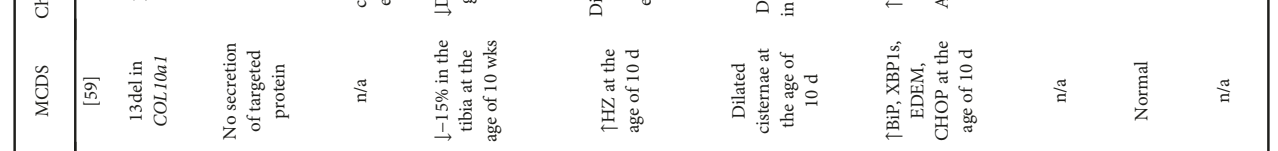

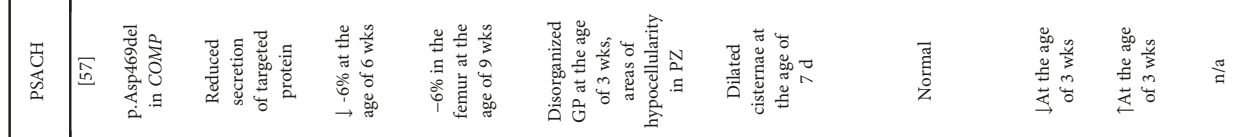

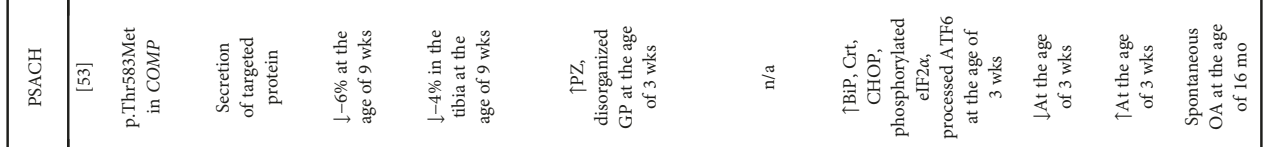

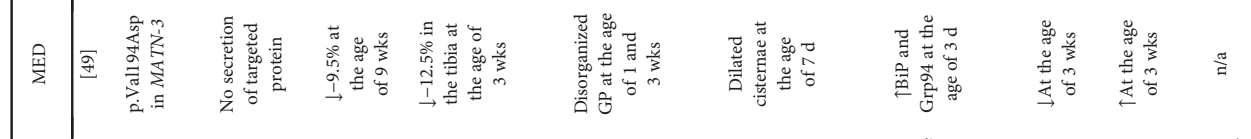

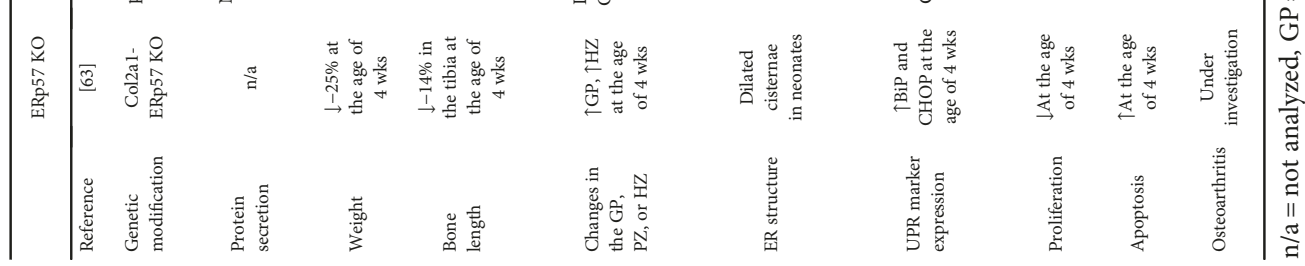


5.1.2. Mouse Model of Spondyloepiphyseal Dysplasia (SED) Associated with a Arg992Cys (p.Arg1192Cys) Mutation in the Col2a1 Gene. Another class of mutations within the human Col2al gene resulting in skeletal abnormalities is a single base substitution that converts codons for arginine in the $\mathrm{Y}$ position of the Gly-X-Y domain to codons for cysteine [44-46]. The most interesting is the SED model associated with an Arg992Cys (p.Arg1192Cys) substitution [45, 46]. These mice reveal ER stress and an altered linear bone growth. The growth plates display a disturbed columnar organization of chondrocytes, an altered collagenous matrix, an atypical cell polarization with unusual organization of primary cilia, and a reduced chondrocyte proliferation. Remarkably, this phenotype can be rescued by switching off the expression of mutated collagen II only during embryonic development or in newborn mice but not later, suggesting that possible therapies in human diseases with this mutation must be applied at prenatal or early postnatal stages in order to be successful [46]. Recently, the impact of arginine-tocysteine mutations in collagen II on protein secretion and cell survival was described elsewhere [47].

\subsubsection{Mouse Model of MED (p.Val194Asp in MATN-3). MED} comprises a range of genetically and phenotypically heterogeneous skeletal dysplasias characterized by disorganized endochondral ossification of the epiphyses of long bones and early-onset osteoarthritis in large weight-bearing joints. Autosomal dominant forms of MED can result from mutations in genes encoding type IX collagen, oligomeric cartilage protein (COMP), and matrilin-3 [36]. The MED-causing mutant proteins are structurally unrelated, but all three comprise perifibrillar constituents and most likely form the structural basis for the mutual interaction of the cartilage fibrils with the extrafibrillar matrix [48]. In addition, the mutations lead at least to partial retention of the affected protein in the ER. Leighton et al. generated a murine model of MED by introducing a specific human disease-causing mutation (p.Val194Asp) into mouse matrilin-3 [49]. Homozygous mice of this genotype develop a progressive chondrodysplasia with weight loss and short-limbed dwarfism. Mutant matrilin-3 is retained within the rough ER of chondrocytes, and the aggregated proteins with aberrant disulfide bonding [50] initiate an unfolded protein response, with upregulation of the UPR marker proteins BiP and calreticulin. The mice display disorganized growth plates with reduced proliferation. In addition, spatially increased apoptosis of chondrocytes occurs. However, whether this chondrocyte death is directly linked to ER stress is unresolved yet, as CHOP expression is not correspondingly augmented $[36,51]$.

\subsubsection{Mouse Models of PSACH (p.Thr583Met and p.D469del} in COMP). Mutations in the cartilage oligomeric protein (COMP) can also cause PSACH. PSACH is a more severe skeletal dysplasia, characterized by a marked short stature, a deformation of the legs, and ligamentous laxity [52]. For a detailed analysis of PSACH, different mouse models were used. One harbors a single point mutation (Thr583Met) in the C-terminal domain of COMP [53] which in patients results in a mild form of the disease with typical radiographic features and waddling gait, but normal or only mild short stature [54]. Mutant mice are normal at birth, but grow slower than their wild-type littermates and also develop a mild short-limbed dwarfism. The chondrocyte columns in the growth plates of these mice are poorly organized. Mutant COMP, however, is secreted into the extracellular matrix, but it is dislocated along with several COMP-binding proteins. Although mutant COMP is not retained within the rough $\mathrm{ER}$, an unfolded protein response with upregulated expression of $\mathrm{BiP}$, calreticulin, phosphorylated eIF $2 \alpha$, and processed ATF6 is initiated. Chondrocyte proliferation is significantly reduced, while apoptosis is both generally increased and spatially dysregulated. By 16 months of age, mutant animals exhibit severe degeneration of articular cartilage, which is consistent with early-onset osteoarthritis seen in PSACH patients [55]. A second transgenic mouse model carries the most frequent mutation in humans, the deletion p.D469del [56]. In these mice, both wild-type and mutant COMP were detected throughout the growth plate. Mutant molecules were restricted to the pericellular matrix, while wild-type COMP showed a uniform distribution throughout the extracellular matrix. Mice expressing the mutant transgene showed a slight gender-specific growth retardation. In mutant animals, the columnar organization in the growth plate was disturbed, proteoglycans were lost, and improperly formed collagen fibrils were observed. In some chondrocytes, the ER was dilated, most probably due to an impaired secretion of mutant COMP similar to that observed in patients. Later in development, the growth plate was irregularly shaped and prematurely invaded by bony tissue [56]. A third mouse model harbors the D469del COMP mutation as a knockin and was generated by homologous recombination [57]. Most phenotypic characteristics were similar to the transgenic mouse, but in contrast, the knockin mouse showed no canonical UPR signaling although proteins aggregated in the ER. Instead, gene profiles of oxidative stress, cell cycle, apoptosis, and NF- $\kappa \mathrm{B}$ signaling changed, suggesting the involvement of UPR-independent stress pathways [57].

5.1.5. Mouse Model of MCDS (13del in COL10a1). MCDS is a dominant disease caused by mutations in the type X collagen gene. Collagen $\mathrm{X}$ is a short, nonfibrillar collagen expressed by hypertrophic chondrocytes in the growth plates of long bones. MCDS patients suffer from a relatively mild chondrodysplasia characterized by growth plate malfunction with a strong expansion of the hypertrophic zones [58]. Almost all patients with MCDS carry mutations in the $\mathrm{NC1}$ domain of type $\mathrm{X}$ collagen [37]. Tsang et al. generated transgenic mice carrying a disease-causing 13 base pair deletion (13del) in this domain [59]. These mice display a chondrodysplasia phenotype including short limbs and expanded hypertrophic zones in growth plates of long bones. Collagen $\mathrm{X}$ is retained in the ER cisternae of hypertrophic chondrocytes, and UPR marker proteins such as $\mathrm{BiP}, \mathrm{XBP} 1 \mathrm{~s}, \mathrm{CHOP}$, and processed ATF6 are upregulated, suggesting MCDS to be an ER stress-related skeletal disease. Furthermore, the authors described changes in the chondrocyte differentiation program as part of the adaptive response to the ER stress. The 
hypertrophic chondrocytes are reprogrammed to a "prehypertrophic chondrocyte-like" cell showing proliferative characteristics to circumvent the expression of mutated collagen X. These aberrations from the normal differentiation processes during endochondral ossification then lead to the chondrodysplasia phenotype [59].

In all of these mouse models, mutations in ECM proteins result in the synthesis of misfolded proteins, which accumulate in the ER and induce the UPR. However, due to the intracellular retention of the mutated proteins, the ECM also lacks essential constituents or contains minor amounts of the affected ECM macromolecules. Therefore, it is hard to determine whether the ER stress itself, the loss of essential ECM components, or both is the underlying mechanism of the given chondrodysplasias.

5.2. Transgenic Mice with Mutations in Genes Encoding Exogenous Proteins That Are Normally Not Expressed in Cartilage. In order to analyze the effects of ER stress on bone development and long bone growth without loss of a single, essential cartilage ECM constituent, transgenic mice were generated in which mutated thyroglobulin $\left(\mathrm{Tg}^{\mathrm{cog}}\right)$ is expressed in chondrocytes $[60,61]$. The mutated thyroglobulin fails to be folded, accumulates in the ER, and induces ER stress. However, as thyroglobulin normally is only expressed in the thyroid gland, the cartilage of these transgenic mouse lines does not lack any specific essential cartilage component.

5.2.1. $\mathrm{Tg}^{\operatorname{cog}}$ Mouse (Col2a1- $\mathrm{Tg}^{\operatorname{cog}}$ ). This mouse line was generated to investigate the generic role of ER stress and the UPR in the pathogenesis of the chondrodysplasia types MED and PSACH. $\mathrm{Tg}^{\mathrm{cog}}$ was expressed as potential ER stress-inducing protein in proliferative chondrocytes under the control of the collagen II alpha 1 promoter. Due to its mutation, $\mathrm{Tg}^{\mathrm{cog}}$ was retained in the ER cisternae, induced ER stress, and activated the UPR. This was detected by increased expression of the ER stress marker protein BiP, phosphorylation of eIF $2 \alpha$, and appearance of XBP1s, the spliced form of XBP1. Col2a1Tg ${ }^{\text {cog }}$ mice displayed diminished long bone growth and a reduced rate of chondrocyte proliferation. However, morphology of the chondrocytes and architecture of the overall growth plate were normal. In addition, no increased apoptosis was detectable. Summarized, these data demonstrate that the targeted induction of ER stress in chondrocytes is sufficient to reduce the rate of bone growth and establishes that classical ER stress is a pathogenic factor that contributes to the disease mechanisms of MED and PSACH. However, as not all pathological features of MED and PSACH were recapitulated, a combination of intra- and extracellular factors is suggested to be responsible for disease pathology [60].

5.2.2. $\mathrm{Tg}^{\mathrm{cog}}$ Mouse (Colloal- $\mathrm{Tg}^{\mathrm{cog}}$ ). An analogous mouse model was established to examine the role of ER stress and the UPR in the pathogenesis of MCDS. Mutant thyroglobulin $\left(\mathrm{Tg}^{\mathrm{cog}}\right)$ was expressed in hypertrophic chondrocytes under the control of the collagen $\mathrm{X}$ promoter. The hypertrophic chondrocytes in these mice exhibited ER stress with a characteristic UPR response. In addition, the hypertrophic zone was expanded, gene expression patterns were disrupted, osteoclast recruitment to the vascular invasion front was reduced, and long bone growth decreased. Moreover, hypertrophic chondrocytes regain a prehypertrophic differentiation state comparable to chondrocytes of the MCDS mice. These data demonstrate that triggering ER stress in hypertrophic chondrocytes per se is sufficient to induce the essential features of the cartilage pathology associated with MCDS and confirm that ER stress is a central pathogenic factor in the disease mechanism [61].

Both $\mathrm{Tg}^{\mathrm{cog}}$ mouse models prove that ER stress is centrally involved in the pathogenesis of chondrodysplasias. The advantage over mouse models with mutant ECM proteins is obvious, but the contribution of a possibly reduced concentration of numerous ECM components cannot clearly be ruled out as a potential factor in the pathogenesis of chondrodysplasias, as the induced UPR reduces the overall protein synthesis in affected chondrocytes. This is the reason why additional mouse models are important to confirm the direct link between ER stress and the pathogenesis of chondrodysplasias.

5.3. Mice with a Knockout of Genes Encoding Proteins of the ER Folding Machinery. To further characterize the role of ER stress as a pathogenic factor in chondrodysplasia, transgenic mice were generated with a cartilage-specific deficiency in proteins of the folding machinery or missing UPR signaling factors.

5.3.1. Hsp47 KO Mouse (Col2a1 Hsp47 KO). The most prominent knockout mouse model in this respect is the cartilagespecific Hsp47 KO mouse [38]. Masago et al. generated a mouse model with a conditionally inactivated Hsp47 gene in chondrocytes using Hsp47 floxed mice and mice carrying a chondrocyte-specific Col2al-Cre transgene. Hsp47 binds Yaa-Gly-Xaa-Arg-Gly in triple-helical procollagen in the ER via hydrophobic and hydrophilic interactions. In cartilage, the binding of $\mathrm{Hsp} 47$ mainly stabilizes procollagen II by preventing unfolding of the triple helix. Thus, Hsp47 is crucial for efficient secretion, processing, fibril formation, and deposition of collagen type II in the ECM of cartilage [62]. These mice die just before or shortly after birth and exhibit a severe generalized chondrodysplasia and bone deformities with lower levels of type II and type XI collagen. Most long bones were severely twisted and shortened. First, these results demonstrate that $\mathrm{Hsp} 47$ is indispensable for well-organized cartilage fibril formation and normal endochondral bone formation. In addition, this mouse model displays numerous characteristics of ER stress. Cartilage collagens and other ECM components accumulated in the rough ER and induced the UPR with induction of the ER stress marker BiP. Moreover, the TUNEL assay revealed an elevated apoptosis rate. This ER stress induced chondrocyte death clearly contributes to the chondrodysplasia phenotype of cartilage-specific Hsp47 KO mice.

5.3.2. ERp57 KO Mouse (Col2a1 ERp57 KO). In our lab, the cartilage-specific ERp57 knockout mouse was intensively investigated [63]. ERp57 is a member of the protein disulfide isomerase family of ER chaperone proteins and is essentially 
involved in the formation of disulfide bridges in newly formed glycoproteins [21]. Moreover, ERp57 accounts for a folding correction in misfolded proteins by elimination of disarranged and formation of new disulfide bridges [64]. The activity of ERp57 is dependent on interactions with calnexin and calreticulin, which mediate the recognition and binding of $\mathrm{N}$-glycosylated substrates [20, 65]. As a total deficiency of ERp57 in mice is lethal at embryonic day 13.5, demonstrating that ERp57 action is indispensable for vertebrate development [66], a cartilage-specific KO mouse model was generated. We used ERp57 floxed mice and crossed these with Col2a1-Cre transgenic animals [63]. The cartilagespecific ERp57 KO animals (ERp57cKO) display an obvious chondrodysplasia-like bone phenotype. Four-week-old male mice reveal a reduced weight, shorter long bones, enlarged growth plates, and a decreased proliferation and increased apoptotic cell death in growth plate chondrocytes. Most likely, this bone phenotype is pronounced especially at this age because of an extremely high protein demand due to the pubertal growth spurt. The significantly reduced tibia lengths and the enlarged growth plates were confirmed by $\mu$-computer tomography analysis. By this method, also altered trabecular structures and a reduced bone volume compared to the total volume were detectable. With respect to the function of ERp57 in protein folding, chondrocytes were analyzed by electron microscopy. Dilated ER structures, which are most likely a consequence of accumulating misfolded proteins and therefore represent a sign of ER stress in these cells, were evident. Immunofluorescence staining of chondrocytes of the tibial growth plate indeed displayed higher amounts of the ER stress markers BiP and CHOP. This demonstrates that ER stress, triggered by the knockout of the protein disulfide isomerase ERp57, is the basic reason of the chondrodysplasia phenotype in these mice [63]. Therefore, mice with a cartilage-specific knockout of the protein disulfide isomerase ERp57 qualify as a novel model for the analysis of ER stress in chondrocytes and ER stress-related skeletal diseases. We wondered that the knockout of this single PDI led to this pronounced phenotype and started to examine ERp57 substrates. In preliminary studies, we observed that the secretion of collagen II by ERp57 KO chondrocytes was almost normal, whereas the proteoglycans were diminished as seen by alcian blue staining of micromasscultured cells. These analyses, however, are far from accurate and should be expanded in future. Jessop et al. analyzed ERp57 substrates by biochemical means and found common structural domains which are important for the interaction of ERp57 with the proteins to be folded [67]. These specific domains may also be one reason why other protein disulfide isomerases, such as PDI or ERp72, could not efficiently compensate for the loss of ERp57. The superior function of the calreticulin-calnexin ERp57 cycle over other protein folding mechanisms [20] might also play a role in this context. The complex of multiple proteins that work together is important for speeding up the folding process. If the calreticulincalnexin-ERp57 cycle fails, this cannot be compensated by individual PDIs. These results and additional detailed analyses of the ERp57 KO mouse in the future will provide access to new insights into ER stress-related cartilage diseases.
As these mice also display chondrodysplasia-like phenotypes, ER stress gets more and more likely as a pathogenic factor in chondrodysplasia.

5.4. Mice with a Knockout of Genes Encoding Proteins Influencing UPR Signaling. ER stress initiates the UPR to restore cellular homeostasis by expression of additional chaperones, reduction of translation, and initiation of the degradation of aggregated proteins. Whenever the UPR fails or is not able to counteract the ER stress, cellular homeostasis cannot be restored and apoptosis is initiated. In mice with deficiencies in UPR signaling factors, this restoration system is reduced, and consequently, the ER stress remains high for a longer period. Therefore, the initiation of pathologic outcomes is anticipated. However, if only one UPR signaling route is blocked, the others probably can substitute for this loss. Here, different mouse models with a failure in ER stress-induced signaling processes in chondrocytes are described exemplarily.

5.4.1. XBP1 KO Mouse (Col2a1-XBP1 KO). In presence of aggregated proteins, the ER sensor protein IRE1 $\alpha$ is activated and then induces splicing of XBP1. The spliced form of XBP1 (XBP1s) acts as a transcription factor inducing the expression of different genes involved in quality control mechanisms of the ER. Cartilage-specific XBP1 knockout mice [39] display a mild form of chondrodysplasia with a delay in endochondral ossification. The main characteristics are dysregulation of chondrocyte proliferation and shortening of hypertrophic growth plate zones. Moreover, long bones reveal a delayed ossification. While ER stress was enhanced in the XBP1-deficient growth plate cartilage and was detectable by IRE1 hyperactivation, only minimal alterations in the expression of chondrocyte proliferation markers were observed and no changes in apoptotic cell death were detectable. The effects of a XBP1 deficiency in cartilage are rather low, but even small imbalances in chondrocytes induce changes in the timing of mineralization during endochondral ossification, ending in an ER stress-induced chondrodysplasia phenotype [39].

5.4.2. ATF4 KO Mouse (Total KO). ATF4 positively regulates the expression of UPR genes that are involved in folding and regulation of autophagy and apoptosis, e.g., CHOP and GADD34 [32]. Compared to XBP1 deficiency, the ablation of Atf4 in mice leads to more severe skeletal defects. ATF4deficient mice display a $50 \%$ reduction in body weight and in femoral bone length at the age of 1 month, indicating a severe limb dwarfism. In the growth plates, the typical columnar structure of proliferative chondrocytes is disturbed and the proliferative zone is shortened. In addition, the hypertrophic zone is abnormally expanded, suggesting a delay in the overall endochondral ossification process. Detailed analysis in chondrocytes showed that ATF4 acts as a transcriptional activator of Indian Hedgehog and therefore controls chondrocyte proliferation and differentiation during bone development and growth [40].

5.4.3. BMP2 KO Mouse (Col2a1-Bmp2 KO). During bone development by endochondral ossification, BMP2 activates 
via Smad-4 the transcription of XBP1, which is involved in ER stress signaling and positively regulates bone formation by activating granulin-epithelin precursor [68]. Both is essential, as mice with a conditional knockout of BMP2 develop a severe chondrodysplasia, with defects in proliferation, differentiation, and apoptosis of chondrocytes in the growth plate [69]. The phenotypes of cartilage-specific BMP2 and XBP1 KO mice are comparable.

5.4.4. S1P KO Mouse (Col2a1-S1P). The site 1 protease is essential in UPR signaling as well, as it is responsible for the processing and activation of the transcription factor ATF6. Due to a loss of active ATF6, one of the three UPR signaling pathways is completely shut down, and therefore, S1P KO mice exhibit a severe chondrodysplasia with a substantial increase in chondrocyte apoptosis. Ultrastructural analysis revealed that the ER in S1P KO chondrocytes displays characteristics of severe ER stress. This suggests that S1P activity is required for the genesis of normal cartilage by endochondral ossification [70].

These mouse models clearly demonstrate that UPR signaling factors play a role not only under pathological but also under physiological conditions in cartilage. Without a well-functioning ER quality control system, normal endochondral ossification and regular bone growth are disturbed.

5.5. Mice with a Knockout of Proteins Involved in the Degradation of Aggregated Proteins. In addition to other processes, the UPR initiates the degradation of aggregated proteins by ER-associated degradation (ERAD type I) or autophagy (ERAD type II). These mechanisms are commenced to liberate the chondrocytes from the overflow of unfolded or misfolded proteins in the ER. However, if the translocation processes into the cytosol or the degradation processes fail, the ER stress in affected chondrocytes remains and the initiation of apoptosis is the last chance to rescue cartilage homeostasis.

5.5.1. Derlin-2 KO (Total KO). A multiprotein complex linking dislocation, ubiquitination, and extraction of misfolded proteins from the ER membrane mediates the dislocation process from the ER to the cytosol in the case that misfolded proteins should be degraded in the proteasome [71]. Derlin-2 is one of the important players within this multiprotein complex. Whole-body deletion of derlin- 2 in mice results in perinatal death of most of the animals due to feeding failures. However, the few mice that survive to adulthood develop a severe chondrodysplasia with defects in ECM protein synthesis and secretion. Derlin-2 KO mice were smaller, had reduced bone lengths, and displayed a striking involution of the rib cages with fusion of the third and fourth sternebrae. Chondrocytes of KO animals showed intracellular retention of ECM components, suggesting a defective protein secretion and degradation. In addition, higher CHOP RNA and protein levels suggest an increase in chondrocyte apoptosis in these animals [72].

5.5.2. CTGF/CCN2 (Total KO). In addition to ERAD type I, autophagy (ERAD type II) is activated in case of ER stress. This mechanism is of great importance for the functionality and homeostasis of the growth plates in mice, as the loss of the connective tissue growth factor (CTGF/CCN2), which is a positive regulator of autophagy, leads to a severe chondrodysplasia. CTGF/CCN2 KO chondrocytes displayed dilated ER cisternae, increased apoptosis, and increased $\mathrm{BiP}$ and CHOP expression levels [73]. Overall, autophagy is considered as a particularly important mechanism for the survival of highly secretory cells under hypoxic and other stressful conditions [74].

The occurrence of chondrodysplasias due to the loss of proteins engaged in ERAD I or II demonstrates the importance of a functional degradation system for unfolded or misfolded proteins. A retention of aggregated proteins in the ER induces ER stress in chondrocytes culminating in dysfunction of the skeletal system.

5.6. Mice with a Knockout of Proteins Which Are Essential for Protein Trafficking and Secretion. In addition to the abovementioned processes, disturbed trafficking of proteins within the cells and to the cell surface and insufficient secretion induce skeletal phenotypes as well.

5.6.1. BBF2H7 KO Mouse (Total KO). Under normal conditions, ER stress induces the activation of the transcription factor BBF2H7, which then, via Sec23, encodes a component that is responsible for the protein transport from the ER to the Golgi apparatus. If BBF2H7 and subsequently Sec23 are absent, the formation of vesicles for these transport processes fails and cartilage matrix secretion is impaired. BBF2H7 KO mice showed severe chondrodysplasia and died by suffocation shortly after birth because of an immature chest cavity. ECM proteins, type II collagen, and COMP remained in the ER; ER stress arose and a severe chondrodysplasia with characteristics, such as a lack of the typical columnar structure in the proliferating zone of growth plates and proliferating chondrocytes showing abnormally expanded ERs developed. This indicates that the ER stress-induced BBF2H7-Sec23a pathway is crucial in endochondral ossification as insufficient protein trafficking into the ECM results in ER stress counteracting proper cartilage proliferation and differentiation processes [75]. In addition, a C-terminal fragment of BBF2H7 accelerates chondrocyte proliferation by binding to Indian hedgehog and its receptor PTCH1, supporting their interaction and signaling [76]. If this acceleration is missing, the proliferative capacity of chondrocytes is reduced.

5.6.2. GMAP-210 KO Mouse (Col2a1-Trip11 KO). Another trafficking component of interest is the golgin Golgi microtubule-associated protein of $210 \mathrm{kDa}$ (GMAP-210). Like other golgins, GMAP-210 is required for efficient trafficking from the ER to the Golgi. Studies by Bird et al. revealed that the skeletal phenotype of achondrogenesis type $1 \mathrm{~A}$ is exclusively caused by defects in chondrocytes, but not osteoblasts, osteoclasts, or hematopoetic cells. Loss of GMAP-210 led to a massive ER swelling and cell death in growth plate cells culminating in impaired bone formation. Notably, the intracellular accumulation of proteins applies only to specific ECM components such as perlecan, but is not a general secretion defect [77]. 

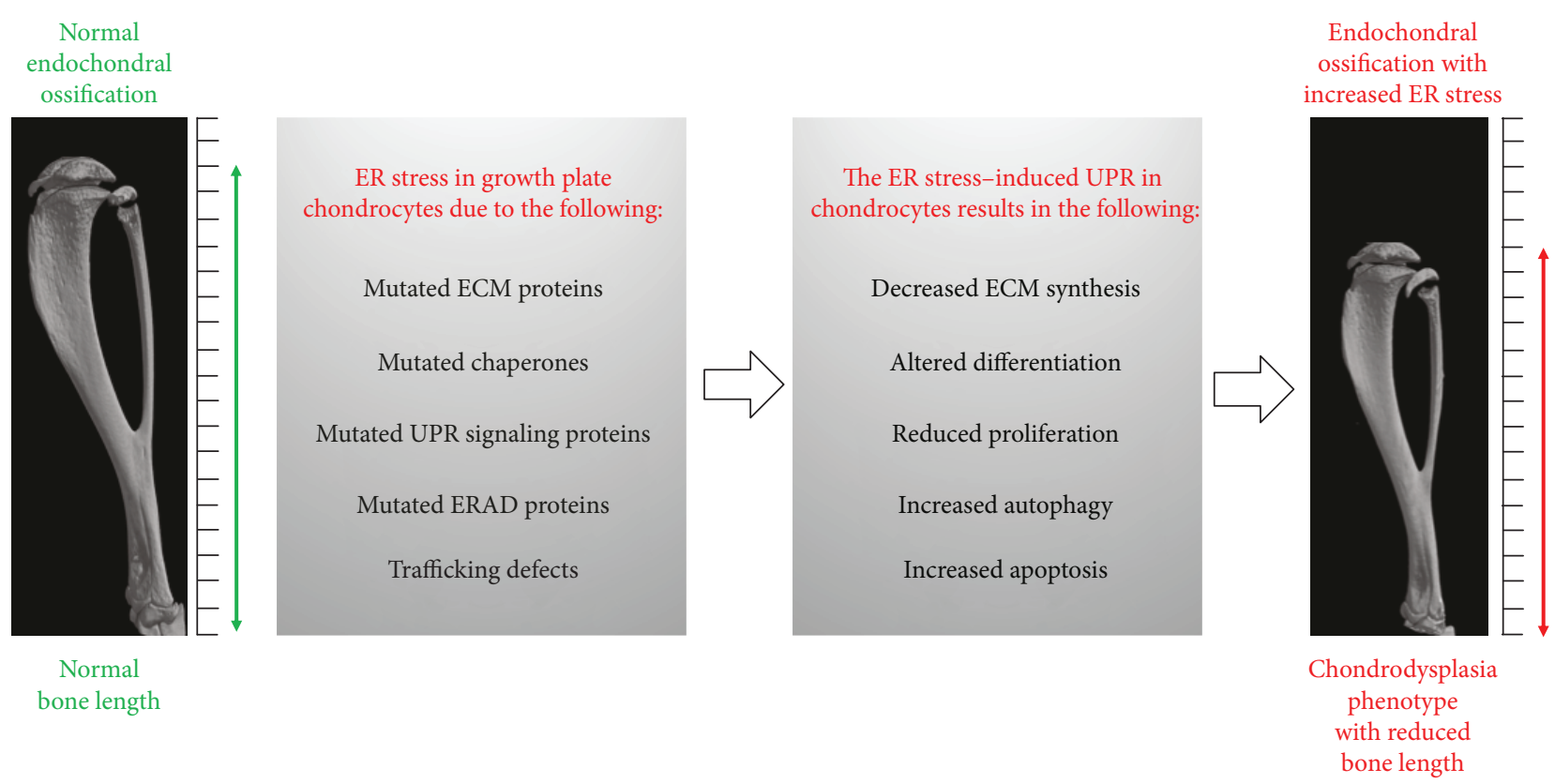

FIGURE 2: Causes and effects of ER stress in growth plate chondrocytes. ER stress in growth plate chondrocytes is induced by mutations in genes encoding ECM components, chaperones, UPR signaling factors, or ERAD proteins. The ER stress-induced unfolded protein response (UPR) substantially impairs essential processes of the endochondral ossification, such as ECM protein synthesis and chondrocyte proliferation and differentiation, and activates autophagy and apoptosis. This results in a chondrodysplasia phenotype with reduced lengths of long bones.

These examples show that mice with trafficking defects display ER stress. Consequently, it seems very likely that chondrodysplasias in mice with such defects are not induced by the loss of nonsecreted components in the ECM, but by the increasing ER stress in chondrocytes.

\section{The Skeletal Phenotypes of Different ER Stress Mouse Models Show a High Degree of Similarity}

Taken together, various mouse models with prolonged or high levels of ER stress in growth plate chondrocytes display chondrodysplasia phenotypes (Figure 2). However, the cause for this pathological outcome varies between the different ER stress mouse models. ER stress may arise due to misfolding of ECM proteins, failures in the ER folding machinery, malfunction of UPR signaling factors, or mutations in proteins involved in the degradation of aggregated proteins. All these cellular dysfunctions result in high levels of ER stress and are obvious, e.g., by dilation of ER cisternae due to the accumulation of unfolded or aggregated proteins. The cells react via UPR by a reduction of protein synthesis, an induction of additional folding enzymes, and by degradation of aggregated proteins. If these rescue mechanisms succeed, chondrocytes regain their normal function. If not, ECM protein synthesis is reduced for a longer period of time, proliferation decreases, differentiation of chondrocytes gets impaired, and apoptosis is augmented. All these changes critically impact bone development.
There are several essential steps in the endochondral ossification process and even slight imbalances at one or the other checkpoint affect the overall process. Long bone growth can be inhibited due to an ER stress-related reduced protein synthesis of cartilage ECM components by affected chondrocytes. The diminished ECM then leads to a reduced bone volume and manifests in a dwarfism disease. Another feature is an ER stress-induced change in chondrocyte proliferation or enhanced apoptosis. Together, this leads to a reduced number of chondrocytes in the growth plates affecting the overall process of endochondral ossification. In addition, ER stress-induced changes in the differentiation program of chondrocytes during endochondral ossification lead to dwarfism diseases. If only one of these necessary events fails, a normal bone development is impossible. This demonstrates that a finely tuned chondrocyte homeostasis with a distinct competence to cope with ER stress in all growth plate zones is essential for a regulated bone development and long bone growth.

\section{ER Stress Also Affects Degenerative Cartilage Diseases Such As Osteoarthritis}

ER stress, however, does not only affect temporary cartilage, which is involved in skeletal development. The function of permanent cartilage in various joints also is dependent on a regulated protein synthesis without enhanced ER stress levels. If this is altered, degenerative cartilage diseases, such as osteoarthritis (OA), may arise. OA is most likely associated with changes in protein synthesis and changes 
in proliferation and differentiation of chondrocytes and enhanced apoptosis. These OA features are similar to ER stress-induced characteristics occurring during bone endochondral ossification.

In general, OA preferentially develops in the elderly. However, chondrodysplasias are often associated with cartilage degradation in young patients [37, 78]. Stickler syndrome, for example, is a dominantly inherited vitreoretinopathy and chondrodysplasia caused by mutations in the genes for types II and XI collagen [79, 80]. About $50 \%$ of all stickler syndrome patients develop severe cartilage erosion in diarthrodial joints before they get 30 years old, and this may be the result of ER stress in articular chondrocytes [81].

In the literature, various studies describe a link between osteoarthritis and ER stress [82-89]. During OA chondrocytes undergo activation, they proliferate and form cell clusters. In addition, the protein synthesis of both ECM molecules and matrix-degrading proteases increases [90], and thus, a high protein load arises in the ER. In late stages of OA, however, cartilage cells reduce their proliferation and die by apoptosis. With age, the expression and activity of folding enzymes for the ER and the UPR signaling decline [91]. Thus, an increased protein synthesis during $\mathrm{OA}$ and a weaker UPR response may induce ER stress, which cannot be compensated [92]. Moreover, the concentration of advanced glycation end products (AGEs) increases and AGEs are known to induce ER stress signaling $[93,94]$. We assume that ER stress may contribute to the development of OA in young chondrodysplasia patients as well as in aged individuals.

In chondrocytes of osteoarthritis patients compared to cartilage cells of healthy controls, a 1.5-fold increase of ERp57 was detected. Simultaneously, calnexin and calreticulin were induced and the stimulation with thapsigargin, an ER stress inducer, increased the ERp57 expression in chondrocytes 2.8-fold [84]. Accordingly, ERp57 and the calnexin/calreticulin cycle appears to be particularly active during ER stress in osteoarthritic cartilage cells. We suggest to employ the cartilage-specific ERp57 KO mouse for a detailed analysis of the contribution of ER stress in the pathogenesis of different skeletal diseases, including OA.

\section{Outlook/Conclusion}

Various mouse models with prolonged or high levels of ER stress in growth plate chondrocytes display chondrodysplasia phenotypes suggesting ER stress as a pathogenic factor contributing to these skeletal diseases. We analyzed in detail the phenotype of the cartilage-specific ERp57 knockout mouse and demonstrated that the deficiency of this single ER-resident protein disulfide isomerase, which is responsible for the correct building of disulfide bridges in cartilage ECM glycoproteins, is sufficient to induce ER stress in chondrocytes and to cause an ER stress-related bone phenotype resembling a chondrodysplasia. This mouse line therefore qualifies as a novel model for the analysis of ER stress in chondrocytes. It may give new insights in ER stress-related short stature disorders and enables the analysis of the role of ER stress in other cartilage diseases, such as osteoarthritis. By proteomic analysis, it would be possible to examine whether specific or all ECM proteins accumulate in the ER of ERp57 cKO mice. Comparable tests in different MED and PSACH mouse models with ER stress revealed genotype-specific differences, suggesting common and distinct disease signatures [95]. This shows that ER stress in different mouse models may have diverse effects. Therefore, multiple ER stress mouse models are valuable to examine cartilage diseases in further detail and to test therapeutics to intervene. Possible therapeutic options are as follows: (1) small molecular chemical chaperones, which efficiently support protein folding in the ER and ameliorate UPR signaling; (2) inhibitors of single UPR pathways, such as PERK signaling inhibitors [96]; (3) activators of ER-associated degradation and autophagy, such as carbamazepine [97]; or (4) inhibitors of excessive apoptosis. However, it is further to be tested whether such compounds alone or in combination are applicable to achieve improvement in ER stress-related cartilage disorders in patients.

\section{Conflicts of Interest}

The authors declare no conflicts of interests.

\section{Acknowledgments}

We would like to thank Uwe Hansen and Tobias Gronau for TEM and $\mu \mathrm{CT}$ analyses of cartilage-specific ERp57 KO mice and Jeannine Wegner for proof reading the manuscript. The work was supported by the German Research Foundation (Grant DR455/3-1 to Rita Dreier).

\section{References}

[1] H. M. Kronenberg, "Developmental regulation of the growth plate," Nature, vol. 423, no. 6937, pp. 332-336, 2003.

[2] E. J. Mackie, L. Tatarczuch, and M. Mirams, "The skeleton: a multi-functional complex organ. The growth plate chondrocyte and endochondral ossification," Journal of Endocrinology, vol. 211, no. 2, pp. 109-121, 2011.

[3] C. Hartmann, "Transcriptional networks controlling skeletal development," Current Opinion in Genetics \& Development, vol. 19, no. 5, pp. 437-443, 2009.

[4] X. Zhou, K. von der Mark, S. Henry, W. Norton, H. Adams, and B. de Crombrugghe, "Chondrocytes transdifferentiate into osteoblasts in endochondral bone during development, postnatal growth and fracture healing in mice," PLoS Genetics, vol. 10, no. 12, article e1004820, 2014.

[5] K. L. Cooper, S. Oh, Y. Sung, R. R. Dasari, M. W. Kirschner, and C. J. Tabin, "Multiple phases of chondrocyte enlargement underlie differences in skeletal proportions," Nature, vol. 495, no. 7441, pp. 375-378, 2013.

[6] E. Zelzer and B. R. Olsen, "The genetic basis for skeletal diseases," Nature, vol. 423, no. 6937, pp. 343-348, 2003.

[7] L. Bonafe, V. Cormier-Daire, C. Hall et al., "Nosology and classification of genetic skeletal disorders: 2015 revision," American Journal of Medical Genetics Part A, vol. 167A, no. 12, pp. 2869-2892, 2015. 
[8] K. Gawron, "Endoplasmic reticulum stress in chondrodysplasias caused by mutations in collagen types II and X," Cell Stress and Chaperones, vol. 21, no. 6, pp. 943-958, 2016.

[9] C. T. Brighton and R. B. Heppenstall, "Oxygen tension in zones of the epiphyseal plate, the metaphysis and diaphysis: an in vitro and in viro study in rats and rabbits," The Journal of Bone \& Joint Surgery, vol. 53, no. 4, pp. 719-728, 1971.

[10] I. A. Silver and A. Maroudas, "Measurement of $\mathrm{pH}$ and ionic composition of pericellular sites," Philosophical Transactions of the Royal Society B: Biological Sciences, vol. 271, no. 912, pp. 261-272, 1975.

[11] S. Hisanaga, M. Miyake, S. Taniuchi et al., "PERK-mediated translational control is required for collagen secretion in chondrocytes," Scientific Reports, vol. 8, no. 1, p. 773, 2018.

[12] M. Wang and R. J. Kaufman, "Protein misfolding in the endoplasmic reticulum as a conduit to human disease," Nature, vol. 529, no. 7586, pp. 326-335, 2016.

[13] G. K. Voeltz, M. M. Rolls, and T. A. Rapoport, "Structural organization of the endoplasmic reticulum," EMBO Reports, vol. 3, no. 10, pp. 944-950, 2002.

[14] L. Halperin, J. Jung, and M. Michalak, "The many functions of the endoplasmic reticulum chaperones and folding enzymes," IUBMB Life, vol. 66, no. 5, pp. 318-326, 2014.

[15] Y. Ishida and K. Nagata, "Hsp47 as a collagen-specific molecular chaperone," Methods in Enzymology, vol. 499, pp. 167-182, 2011.

[16] P. E. Shaw, "Peptidyl-prolyl isomerases: a new twist to transcription," EMBO Reports, vol. 3, no. 6, pp. 521-526, 2002.

[17] M. Aebi, R. Bernasconi, S. Clerc, and M. Molinari, "N-glycan structures: recognition and processing in the ER," Trends in Biochemical Sciences, vol. 35, no. 2, pp. 74-82, 2010.

[18] J. J. Caramelo and A. J. Parodi, "Getting in and out from calnexin/calreticulin cycles," Journal of Biological Chemistry, vol. 283, no. 16, pp. 10221-10225, 2008.

[19] K. McCaffrey and I. Braakman, "Protein quality control at the endoplasmic reticulum," Essays in Biochemistry, vol. 60, no. 2, pp. 227-235, 2016.

[20] C. E. Jessop, T. J. Tavender, R. H. Watkins, J. E. Chambers, and N. J. Bulleid, "Substrate specificity of the oxidoreductase ERp57 is determined primarily by its interaction with calnexin and calreticulin," Journal of Biological Chemistry, vol. 284, no. 4, pp. 2194-2202, 2009.

[21] J. D. Oliver, F. J. Van der Wal, N. J. Bulleid, and S. High, "Interaction of the thiol-dependent reductase ERp57 with nascent glycoproteins," Science, vol. 275, no. 5296, pp. 86-88, 1997.

[22] A. Hettinghouse, R. Liu, and C. J. Liu, "Multifunctional molecule ERp57: from cancer to neurodegenerative diseases," Pharmacology \& Therapeutics, vol. 181, pp. 34-48, 2018.

[23] E. Fujita, Y. Kouroku, A. Isoai et al., "Two endoplasmic reticulum-associated degradation (ERAD) systems for the novel variant of the mutant dysferlin: ubiquitin/proteasome ERAD(I) and autophagy/lysosome ERAD(II)," Human Molecular Genetics, vol. 16, no. 6, pp. 618-629, 2007.

[24] R. J. Kaufman, "Orchestrating the unfolded protein response in health and disease," The Journal of Clinical Investigation, vol. 110, no. 10, pp. 1389-1398, 2002.

[25] C. Hetz, "The unfolded protein response: controlling cell fate decisions under ER stress and beyond," Nature Reviews Molecular Cell Biology, vol. 13, no. 2, pp. 89-102, 2012.

[26] Y. Kozutsumi, M. Segal, K. Normington, M. J. Gething, and J. Sambrook, "The presence of malfolded proteins in the endoplasmic reticulum signals the induction of glucoseregulated proteins," Nature, vol. 332, no. 6163, pp. 462-464, 1988.

[27] P. Walter and D. Ron, "The unfolded protein response: from stress pathway to homeostatic regulation," Science, vol. 334, no. 6059, pp. 1081-1086, 2011.

[28] K. Haze, H. Yoshida, H. Yanagi, T. Yura, and K. Mori, “Mammalian transcription factor ATF6 is synthesized as a transmembrane protein and activated by proteolysis in response to endoplasmic reticulum stress," Molecular Biology of the Cell, vol. 10, no. 11, pp. 3787-3799, 1999.

[29] C. Y. Liu, M. Schroder, and R. J. Kaufman, "Ligand-independent dimerization activates the stress response kinases IRE1 and PERK in the lumen of the endoplasmic reticulum," Journal of Biological Chemistry, vol. 275, no. 32, pp. 2488124885, 2000.

[30] J. Hollien and J. S. Weissman, "Decay of endoplasmic reticulum-localized mRNAs during the unfolded protein response," Science, vol. 313, no. 5783, pp. 104-107, 2006.

[31] H. P. Harding, Y. Zhang, A. Bertolotti, H. Zeng, and D. Ron, "Perk is essential for translational regulation and cell survival during the unfolded protein response," Molecular Cell, vol. 5, no. 5, pp. 897-904, 2000.

[32] H. P. Harding, I. Novoa, Y. Zhang et al., "Regulated translation initiation controls stress-induced gene expression in mammalian cells," Molecular Cell, vol. 6, no. 5, pp. 1099 $1108,2000$.

[33] K. Hata, Y. Takahata, T. Murakami, and R. Nishimura, "Transcriptional network controlling endochondral ossification," Journal of Bone Metabolism, vol. 24, no. 2, pp. 75-82, 2017.

[34] X. Kang, W. Yang, D. Feng et al., "Cartilage-specific autophagy deficiency promotes ER stress and impairs chondrogenesis in PERK-ATF4-CHOP-dependent manner," Journal of Bone and Mineral Research, vol. 32, no. 10, pp. 2128-2141, 2017.

[35] M. Ni and A. S. Lee, "ER chaperones in mammalian development and human diseases," FEBS Letters, vol. 581, no. 19, pp. 3641-3651, 2007.

[36] R. P. Boot-Handford and M. D. Briggs, "The unfolded protein response and its relevance to connective tissue diseases," Cell and Tissue Research, vol. 339, no. 1, pp. 197-211, 2010.

[37] S. E. Patterson and C. N. Dealy, "Mechanisms and models of endoplasmic reticulum stress in chondrodysplasia," Developmental Dynamics, vol. 243, no. 7, pp. 875-893, 2014.

[38] Y. Masago, A. Hosoya, K. Kawasaki et al., "The molecular chaperone Hsp47 is essential for cartilage and endochondral bone formation," Journal of Cell Science, vol. 125, no. 5, pp. 1118-1128, 2012.

[39] T. L. Cameron, I. L. Gresshoff, K. M. Bell et al., "Cartilagespecific ablation of XBP1 signaling in mouse results in a chondrodysplasia characterized by reduced chondrocyte proliferation and delayed cartilage maturation and mineralization," Osteoarthritis and Cartilage, vol. 23, no. 4, pp. 661-670, 2015.

[40] W. Wang, N. Lian, L. Li et al., "Atf4 regulates chondrocyte proliferation and differentiation during endochondral ossification by activating Ihh transcription," Development, vol. 136, no. 24, pp. 4143-4153, 2009.

[41] C. Gentili and R. Cancedda, "Cartilage and bone extracellular matrix," Current Pharmaceutical Design, vol. 15, no. 12, pp. 1334-1348, 2009. 
[42] P. Kannu, J. Bateman, and R. Savarirayan, "Clinical phenotypes associated with type II collagen mutations," Journal of Paediatrics and Child Health, vol. 48, no. 2, pp. E38-E43, 2012.

[43] G. Liang, C. Lian, D. Huang et al., "Endoplasmic reticulum stress-unfolding protein response-apoptosis cascade causes chondrodysplasia in a col2a1 p.Gly1170Ser mutated mouse model," PLoS One, vol. 9, no. 1, article e86894, 2014.

[44] M. Arita, S. W. Li, G. Kopen, E. Adachi, S. A. Jimenez, and A. Fertala, "Skeletal abnormalities and ultrastructural changes of cartilage in transgenic mice expressing a collagen II gene (COL2A1) with a Cys for Arg- $\alpha 1-519$ substitution," Osteoarthritis and Cartilage, vol. 10, no. 10, pp. 808-815, 2002.

[45] M. Arita, J. Fertala, C. Hou, A. Steplewski, and A. Fertala, "Mechanisms of aberrant organization of growth plates in conditional transgenic mouse model of spondyloepiphyseal dysplasia associated with the R992C substitution in collagen II," The American Journal of Pathology, vol. 185, no. 1, pp. 214-229, 2015.

[46] J. Fertala, M. Arita, A. Steplewski, W. V. Arnold, and A. Fertala, "Epiphyseal growth plate architecture is unaffected by early postnatal activation of the expression of R992C collagen II mutant," Bone, vol. 112, pp. 42-50, 2018.

[47] S. A. Chakkalakal, J. Heilig, U. Baumann, M. Paulsson, and F. Zaucke, "Impact of arginine to cysteine mutations in collagen II on protein secretion and cell survival," International Journal of Molecular Sciences, vol. 19, no. 2, 2018.

[48] B. Budde, K. Blumbach, J. Ylostalo et al., "Altered integration of matrilin-3 into cartilage extracellular matrix in the absence of collagen IX," Molecular and Cellular Biology, vol. 25, no. 23, pp. 10465-10478, 2005.

[49] M. P. Leighton, S. Nundlall, T. Starborg et al., "Decreased chondrocyte proliferation and dysregulated apoptosis in the cartilage growth plate are key features of a murine model of epiphyseal dysplasia caused by a matn3 mutation," Human Molecular Genetics, vol. 16, no. 14, pp. 1728-1741, 2007.

[50] C. L. Hartley, S. Edwards, L. Mullan et al., "Armet/Manf and Creld2 are components of a specialized ER stress response provoked by inappropriate formation of disulphide bonds: implications for genetic skeletal diseases," Human Molecular Genetics, vol. 22, no. 25, pp. 5262-5275, 2013.

[51] S. Nundlall, M. H. Rajpar, P. A. Bell et al., "An unfolded protein response is the initial cellular response to the expression of mutant matrilin-3 in a mouse model of multiple epiphyseal dysplasia," Cell Stress and Chaperones, vol. 15, no. 6, pp. 835849, 2010.

[52] M. D. Briggs and K. L. Chapman, "Pseudoachondroplasia and multiple epiphyseal dysplasia: mutation review, molecular interactions, and genotype to phenotype correlations," Human Mutation, vol. 19, no. 5, pp. 465-478, 2002.

[53] K. A. Pirog-Garcia, R. S. Meadows, L. Knowles et al., "Reduced cell proliferation and increased apoptosis are significant pathological mechanisms in a murine model of mild pseudoachondroplasia resulting from a mutation in the C-terminal domain of COMP," Human Molecular Genetics, vol. 16, no. 17, pp. 2072-2088, 2007.

[54] M. D. Briggs, G. R. Mortier, W. G. Cole et al., "Diverse mutations in the gene for cartilage oligomeric matrix protein in the pseudoachondroplasia-multiple epiphyseal dysplasia disease spectrum," American Journal of Human Genetics, vol. 62, no. 2, pp. 311-319, 1998.

[55] K. A. Pirog, A. Irman, S. Young et al., "Abnormal chondrocyte apoptosis in the cartilage growth plate is influenced by genetic background and deletion of CHOP in a targeted mouse model of pseudoachondroplasia," PLoS One, vol. 9, no. 2, article e85145, 2014.

[56] M. Schmitz, A. Niehoff, N. Miosge, N. Smyth, M. Paulsson, and F. Zaucke, "Transgenic mice expressing D469 $\Delta$ mutated cartilage oligomeric matrix protein (COMP) show growth plate abnormalities and sternal malformations," Matrix Biology, vol. 27, no. 2, pp. 67-85, 2008.

[57] F. Suleman, B. Gualeni, H. J. Gregson et al., "A novel form of chondrocyte stress is triggered by a COMP mutation causing pseudoachondroplasia," Human Mutation, vol. 33, no. 1, pp. 218-231, 2012.

[58] M. S. P. Ho, K. Y. Tsang, R. L. K. Lo et al., "COL10A1 nonsense and frame-shift mutations have a gain-of-function effect on the growth plate in human and mouse metaphyseal chondrodysplasia type Schmid," Human Molecular Genetics, vol. 16, no. 10, pp. 1201-1215, 2007.

[59] K. Y. Tsang, D. Chan, D. Cheslett et al., "Surviving endoplasmic reticulum stress is coupled to altered chondrocyte differentiation and function," PLoS Biology, vol. 5, no. 3, article e44, 2007.

[60] L. H. W. Kung, M. H. Rajpar, R. Preziosi, M. D. Briggs, and R. P. Boot-Handford, "Increased classical endoplasmic reticulum stress is sufficient to reduce chondrocyte proliferation rate in the growth plate and decrease bone growth," PLoS One, vol. 10, no. 2, article e0117016, 2015.

[61] M. H. Rajpar, B. McDermott, L. Kung et al., "Targeted induction of endoplasmic reticulum stress induces cartilage pathology," PLoS Genetics, vol. 5, no. 10, article e1000691, 2009.

[62] S. Ito and K. Nagata, "Biology of Hsp47 (serpin H1), a collagen-specific molecular chaperone," Seminars in Cell \& Developmental Biology, vol. 62, pp. 142-151, 2017.

[63] A. Linz, Y. Knieper, T. Gronau et al., "ER stress during the pubertal growth spurt results in impaired long-bone growth in chondrocyte-specific ERp57 knockout mice," Journal of Bone and Mineral Research, vol. 30, no. 8, pp. 1481-1493, 2015.

[64] L. Ellgaard and L. W. Ruddock, "The human protein disulphide isomerase family: substrate interactions and functional properties," EMBO Reports, vol. 6, no. 1, pp. 28-32, 2005.

[65] J. D. Oliver, H. L. Roderick, D. H. Llewellyn, and S. High, "ERp57 functions as a subunit of specific complexes formed with the ER lectins calreticulin and calnexin," Molecular Biology of the Cell, vol. 10, no. 8, pp. 2573-2582, 1999.

[66] H. Coe, J. Jung, J. Groenendyk, D. Prins, and M. Michalak, "ERp57 modulates STAT3 signaling from the lumen of the endoplasmic reticulum," Journal of Biological Chemistry, vol. 285, no. 9, pp. 6725-6738, 2010.

[67] C. E. Jessop, S. Chakravarthi, N. Garbi, G. J. Hammerling, S. Lovell, and N. J. Bulleid, "ERp57 is essential for efficient folding of glycoproteins sharing common structural domains," The EMBO Journal, vol. 26, no. 1, pp. 28-40, 2007.

[68] F. J. Guo, Z. Xiong, X. Han et al., "XBP1S, a BMP2-inducible transcription factor, accelerates endochondral bone growth by activating GEP growth factor," Journal of Cellular and Molecular Medicine, vol. 18, no. 6, pp. 1157-1171, 2014.

[69] B. Shu, M. Zhang, R. Xie et al., "BMP2, but not BMP4, is crucial for chondrocyte proliferation and maturation during endochondral bone development," Journal of Cell Science, vol. 124, no. 20, pp. 3428-3440, 2011. 
[70] D. Patra, X. Xing, S. Davies et al., "Site-1 protease is essential for endochondral bone formation in mice," The Journal of Cell Biology, vol. 179, no. 4, pp. 687-700, 2007.

[71] B. N. Lilley and H. L. Ploegh, "Multiprotein complexes that link dislocation, ubiquitination, and extraction of misfolded proteins from the endoplasmic reticulum membrane," Proceedings of the National Academy of Sciences of the United States of America, vol. 102, no. 40, pp. 14296-14301, 2005.

[72] S. K. Dougan, C. C. A. Hu, M. E. Paquet et al., "Derlin-2-deficient mice reveal an essential role for protein dislocation in chondrocytes," Molecular and Cellular Biology, vol. 31, no. 6, pp. 1145-1159, 2011.

[73] F. Hall-Glenn, A. Aivazi, L. Akopyan et al., "CCN2/CTGF is required for matrix organization and to protect growth plate chondrocytes from cellular stress," Journal of Cell Communication and Signaling, vol. 7, no. 3, pp. 219-230, 2013.

[74] V. Srinivas, J. Bohensky, A. M. Zahm, and I. M. Shapiro, "Autophagy in mineralizing tissues: microenvironmental perspectives," Cell Cycle, vol. 8, no. 3, pp. 391-393, 2009.

[75] A. Saito, S. Hino, T. Murakami et al., "Regulation of endoplasmic reticulum stress response by a $\mathrm{BBF} 2 \mathrm{H} 7$-mediated Sec23a pathway is essential for chondrogenesis," Nature Cell Biology, vol. 11, no. 10, pp. 1197-1204, 2009.

[76] A. Saito, S. Kanemoto, Y. Zhang, R. Asada, K. Hino, and K. Imaizumi, "Chondrocyte proliferation regulated by secreted luminal domain of ER stress transducer BBF2H7/CREB3L2," Molecular Cell, vol. 53, no. 1, pp. 127-139, 2014.

[77] I. M. Bird, S. H. Kim, D. K. Schweppe et al., "The skeletal phenotype of achondrogenesis type $1 \mathrm{~A}$ is caused exclusively by cartilage defects," Development, vol. 145, no. 1, 2018.

[78] A. Hughes, A. E. Oxford, K. Tawara, C. L. Jorcyk, and J. T. Oxford, "Endoplasmic reticulum stress and unfolded protein response in cartilage pathophysiology; contributing factors to apoptosis and osteoarthritis," International Journal of Molecular Sciences, vol. 18, no. 3, 2017.

[79] N. N. Ahmad, L. Ala-Kokko, R. G. Knowlton et al., "Stop codon in the procollagen II gene (COL2A1) in a family with the Stickler syndrome (arthro-ophthalmopathy)," Proceedings of the National Academy of Sciences of the United States of America, vol. 88, no. 15, pp. 6624-6627, 1991.

[80] A. J. Richards, J. R. Yates, R. Williams et al., "A family with Stickler syndrome type 2 has a mutation in the COL11A1 gene resulting in the substitution of glycine 97 by valine in a1(XI) collagen," Human Molecular Genetics, vol. 5, no. 9, pp. 1339-1343, 1996.

[81] T. Couchouron and C. Masson, "Early-onset progressive osteoarthritis with hereditary progressive ophtalmopathy or Stickler syndrome," Joint, Bone, Spine, vol. 78, no. 1, pp. 4549, 2011.

[82] K. Horiuchi, T. Tohmonda, and H. Morioka, "The unfolded protein response in skeletal development and homeostasis," Cellular and Molecular Life Sciences, vol. 73, no. 15, pp. 2851-2869, 2016.

[83] A. Hosseinzadeh, S. K. Kamrava, M. T. Joghataei et al., “Apoptosis signaling pathways in osteoarthritis and possible protective role of melatonin," Journal of Pineal Research, vol. 61, no. 4, pp. 411-425, 2016.

[84] Y. H. Li, G. Tardif, D. Hum et al., "The unfolded protein response genes in human osteoarthritic chondrocytes: PERK emerges as a potential therapeutic target," Arthritis Research \& Therapy, vol. 18, no. 1, p. 172, 2016.
[85] R. Liu-Bryan and R. Terkeltaub, "Emerging regulators of the inflammatory process in osteoarthritis," Nature Reviews Rheumatology, vol. 11, no. 1, pp. 35-44, 2015.

[86] A. E. Nugent, D. M. Speicher, I. Gradisar et al., "Advanced osteoarthritis in humans is associated with altered collagen VI expression and upregulation of ER-stress markers Grp78 and bag-1," Journal of Histochemistry \& Cytochemistry, vol. 57, no. 10, pp. 923-931, 2009.

[87] K. Takada, J. Hirose, K. Senba et al., "Enhanced apoptotic and reduced protective response in chondrocytes following endoplasmic reticulum stress in osteoarthritic cartilage," International Journal of Experimental Pathology, vol. 92, no. 4, pp. 232-242, 2011.

[88] Y. Uehara, J. Hirose, S. Yamabe et al., "Endoplasmic reticulum stress-induced apoptosis contributes to articular cartilage degeneration via C/EBP homologous protein," Osteoarthritis and Cartilage, vol. 22, no. 7, pp. 1007-1017, 2014.

[89] X. Yuan, H. Liu, L. Li et al., "The roles of endoplasmic reticulum stress in the pathophysiological development of cartilage and chondrocytes," Current Pharmaceutical Design, vol. 23, no. 11, pp. 1693-1704, 2017.

[90] M. B. Goldring and K. B. Marcu, "Cartilage homeostasis in health and rheumatic diseases," Arthritis Research \& Therapy, vol. 11, no. 3, p. 224, 2009.

[91] M. K. Brown and N. Naidoo, "The endoplasmic reticulum stress response in aging and age-related diseases," Frontiers in Physiology, vol. 3, p. 263, 2012.

[92] C. Lopez-Otin, M. A. Blasco, L. Partridge, M. Serrano, and G. Kroemer, "The hallmarks of aging," Cell, vol. 153, no. 6, pp. 1194-1217, 2013.

[93] C. Adamopoulos, E. Farmaki, E. Spilioti, H. Kiaris, C. Piperi, and A. G. Papavassiliou, "Advanced glycation end-products induce endoplasmic reticulum stress in human aortic endothelial cells," Clinical Chemistry and Laboratory Medicine, vol. 52, no. 1, pp. 151-160, 2014.

[94] S. Yamabe, J. Hirose, Y. Uehara et al., "Intracellular accumulation of advanced glycation end products induces apoptosis via endoplasmic reticulum stress in chondrocytes," FEBS Journal, vol. 280, no. 7, pp. 1617-1629, 2013.

[95] P. A. Bell, R. Wagener, F. Zaucke et al., "Analysis of the cartilage proteome from three different mouse models of genetic skeletal diseases reveals common and discrete disease signatures," Biology Open, vol. 2, no. 8, pp. 802-811, 2013.

[96] C. Wang, Z. Tan, B. Niu et al., "Inhibiting the integrated stress response pathway prevents aberrant chondrocyte differentiation thereby alleviating chondrodysplasia," eLife, vol. 7, 2018.

[97] L. A. Mullan, E. J. Mularczyk, L. H. Kung et al., "Increased intracellular proteolysis reduces disease severity in an ER stress-associated dwarfism," The Journal of Clinical Investigation, vol. 127, no. 10, pp. 3861-3865, 2017. 


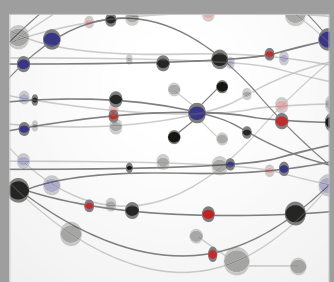

The Scientific World Journal
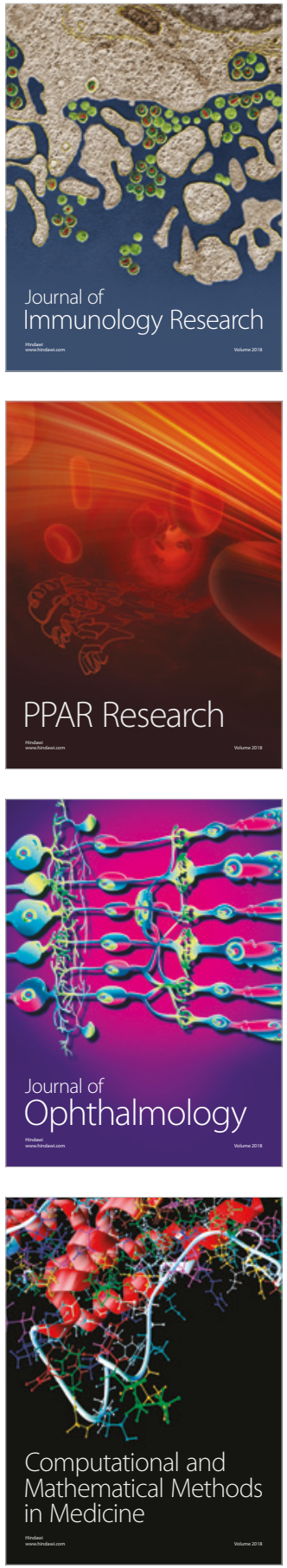

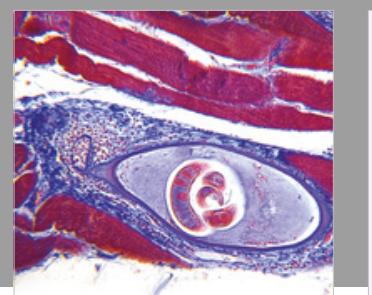

Gastroenterology Research and Practice

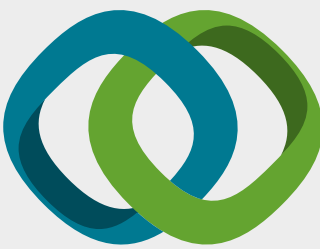

\section{Hindawi}

Submit your manuscripts at

www.hindawi.com
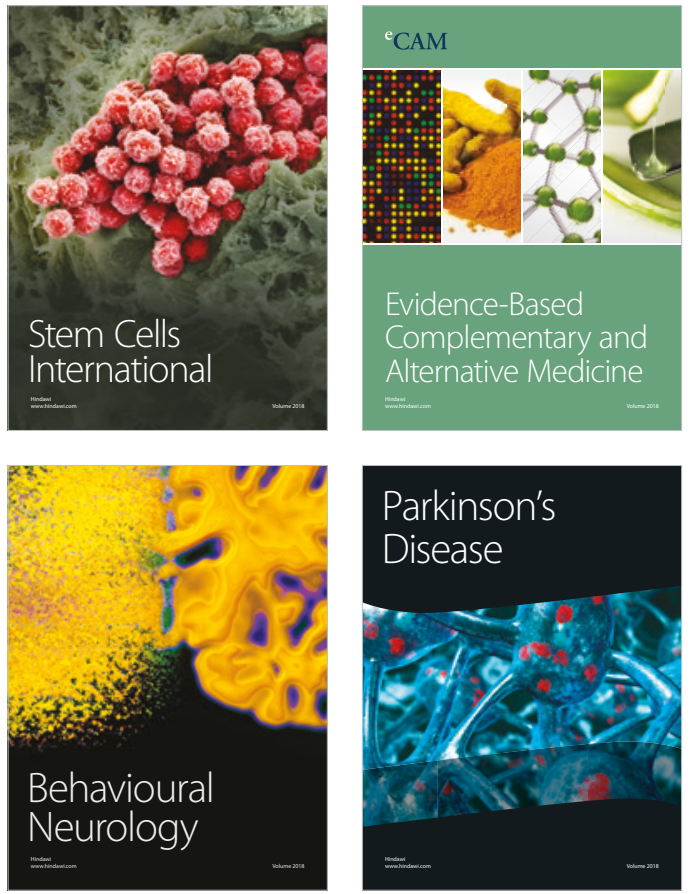

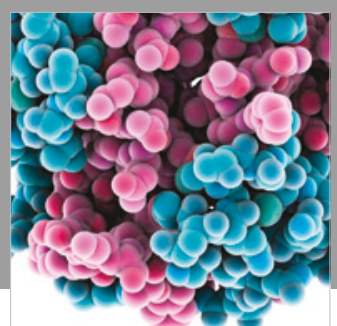

ournal of

Diabetes Research

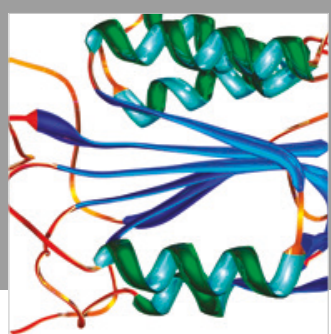

Disease Markers
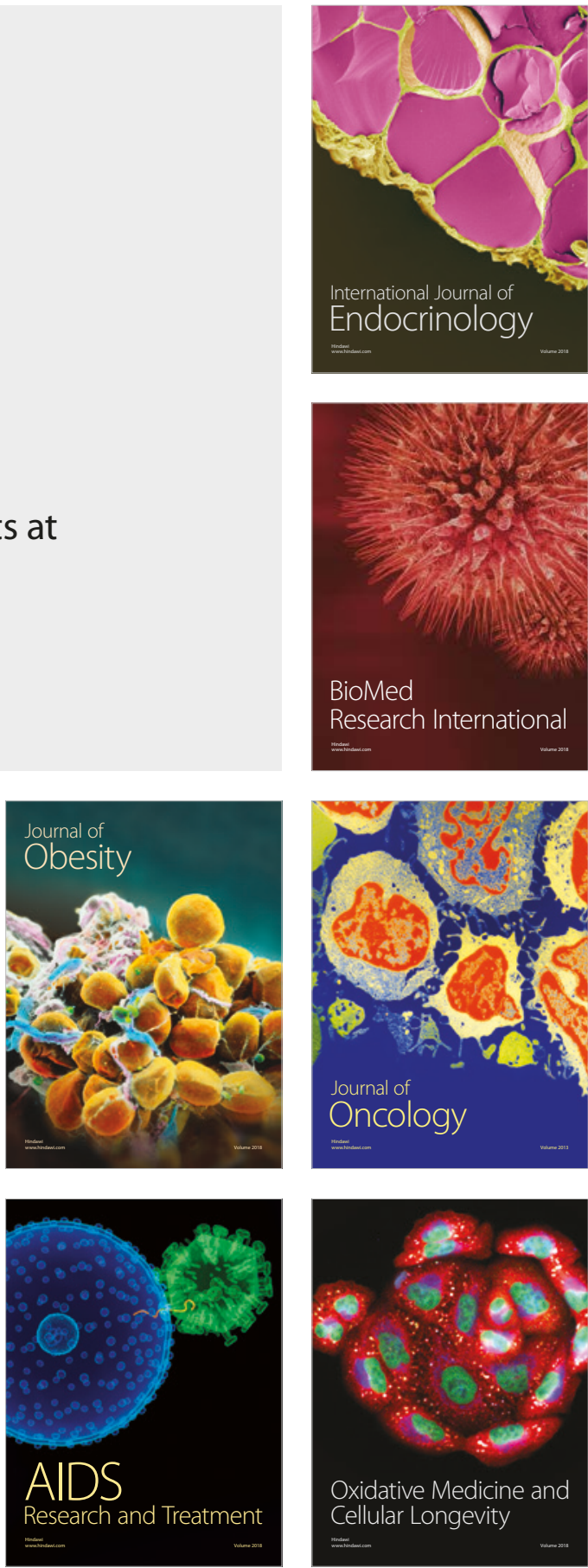